\title{
Posterior reversible encephalopathy syndrome: a rare case with delayed reverse
}

\author{
Islam AT ${ }^{\mathrm{a}}$, Uddin $\mathrm{MK}^{\mathrm{b}}$, Ali MA ${ }^{\mathrm{b}}$, Kundu $\mathrm{PK}^{\mathrm{b}}$, Alahi MMc, Amin MPc ${ }^{\mathrm{c}}$, Sarkar MK ${ }^{\mathrm{c}}$
}

\begin{abstract}
Posterior reversible encephalopathy syndrome (PRES) is a reversible phenomenon diagnosed with both clinical and radiological criteria. We present anatypical case of PRES with unusual delayed reversal. A 29-year-old female presented withseizure and altered consciousness during post-partum periods. The initial computed tomographyscan of brain was normal. But subsequent magnetic resonance imaging of brain showed hyperintense lesion in rightparieto-occipital region. After 7 weeks of multidisciplinary team effort, the patient recovered both clinically and radiologically. High index of suspicion with long term follow up is crucial for this curable and reversible syndrome. Because of rarity in delayed recovery, this case of PRES is reported.
\end{abstract}

Keywords: Posterior reversible encephalopathy syndrome, vasogenic edema,delayed recovery.

(BIRDEM Med J 2019; 9(3): 248-250)

\section{Introduction}

Posterior reversible encephalopathy syndrome (PRES) is a clinical-radiological diagnosis whichis often under diagnosed or misdiagnosed due to rarity of the disease. PRES manifests clinically with headache, visual disturbance, seizure or decreased consciousness. ${ }^{1}$ Radiologically, cerebral edema at parietal and occipital lobes is diagnostic for PRES. ${ }^{1}$ Vasogenic edema in the posterior cerebral circulation region is the hallmark for the diagnosis of PRES. ${ }^{1}$ The commonest etiology for PRES is eclampsia. ${ }^{2,3}$ Post-partum eclampsia is also a common cause for PRES and the average duration from delivery to hospital admission due to PRES is 6.9 days. $^{3}$ Subjective improvement occurs within few weeks and improvement of imaging may require several weeks. ${ }^{3}$

\section{Author information}

a. Ahmed Tanjimul Islam, Medical Officer of Neurology, Rajshahi Medical College Hospital, (RMCH), Rajshahi, Bangladesh.

b. Md. Kafil Uddin, Md. Ahmed Ali, Pijush Kumar Kundu, Associate Professor of Neurology, Rajshahi Medical College Hospital, (RMCH), Rajshahi, Bangladesh.

c. Md. MunzurAlahi, Md. Parvez Amin, Mukul Kumar Sarkar, Assistant Professor of Neurology, Rajshahi Medical College Hospital, (RMCH), Rajshahi, Bangladesh.

Address of correspondence: Ahmed Tanjimul Islam, MSc (Leeds, England), MD, Medical Officer of Neurology, Rajshahi Medical College Hospital (RMCH), Rajshahi, Bangladesh. Email: droveesomch@gmail.com

Received: March 4, 2019

Accepted: July 31, 2019
Due to its reversibility and complete recovery, it is important to diagnose and treat PRES cases in the early stages. Previously very few cases were reported as post-partum PRES . ${ }^{3-5}$ Again, to the best of our knowledge, only one case had been reported with an unusual delayed recovery from PRES due to co-morbid conditions (acute renal failure with electrolyte imbalance). ${ }^{4}$ Therefore, we present this rare case of PRES with delayed reversal.

\section{Case report}

A 29-year-old normotensive, non-diabetic lady in her post-partum period presented with seizure and impaired consciousness. Seizure was sudden onset, generalized tonic-clonic, single episode followed by loss of consciousness. On admission at emergency department, the Glasgow Coma Scale (GCS) was 6 (E1V2M3) $(\mathrm{E}=$ eye opening, $\mathrm{V}=$ verbal Response, $\mathrm{M}=$ motor response), pupil was normal-reactive and symmetrical, respiration was normal and blood pressure (BP) was 210/110 mm $\mathrm{Hg}$. On neurological examination, there was equivocalbilateral planter response. Bilateral papilledema (Grade 2) was found onophthalmoscopic examination. Other systemic examination revealed no abnormality.

Initial routine investigation revealed mild anemia (hemoglobin $10.1 \mathrm{gm} / \mathrm{dl}$ ), slightly raisedserum 
creatinine $(1.4 \mathrm{mg} / \mathrm{dl})$, hyperglycemia (random blood glucose $11.4 \mathrm{mmol} / \mathrm{l}$ ) andhyponatremia (serum sodium $130 \mathrm{meq} / \mathrm{l})$. Immediate computed tomography (CT) scan of brain appeared normal. Magnetic resonance imaging (MRI) of brain obtained after 18 hours revealed hyperintense lesion in the bilateral occipital lobes (Figure 1). Diffusion weighted imaging (DWI) showed restricted diffusion in occipital region.

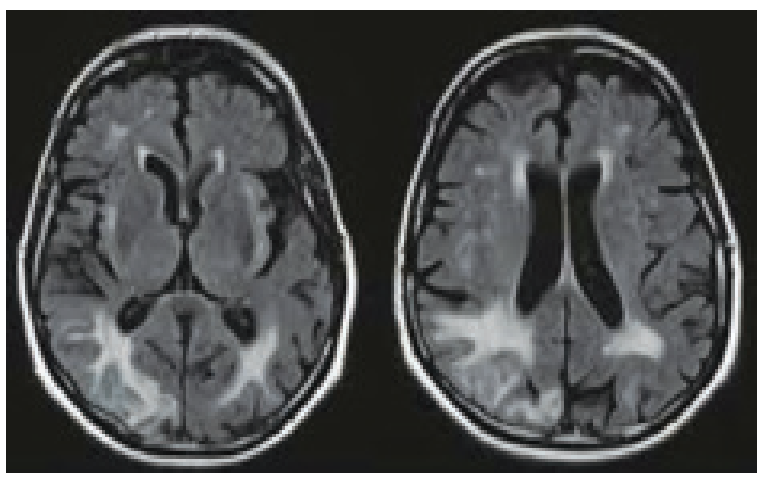

Figure 1 MRI of brain (FLAIR) showing hyper-intense lesion in parieto-occipital region (after 18 hours of admission)

The patient was diagnosed and treated as hypertensive encephalopathy. The management goal was to treat the hypertension, hyperglycemia, electrolyte imbalance and prevention of complications. The management was continued by transferring the patient to a higher center where she was managed in high dependency unit (HDU) with oral combined anti-hypertensive drugs (amlodipine $5 \mathrm{mg}$ and olmesartan $20 \mathrm{mg}$ daily through nasogastric tube)and continuous monitoring of intake output, blood glucose and vital signs. Regular follow up was given with correction of hyperglycemia, electrolyte imbalance and pulmonary infection. The medical team (Neurologist, Internist and Physiotherapist) decided to continue the treatment in HDU setting. At the $4^{\text {th }}$ week, the patient developed aspiration pneumonia and pressure sore (sacral) which was diagnosed by routine blood tests, chest x-ray and culture (blood and pus) and managed successfully (antibiotics and regular dressing). There was no improvement of GCS (less than 8) during the first 6 weeks.

The GCS of the patient started to improve gradually at $7^{\text {th }}$ week of her admission. The GCS was found 8 $(\mathrm{E} 1 \mathrm{~V} 3 \mathrm{M} 4)$ at $^{\text {th }}$ week and $11(\mathrm{E} 2 \mathrm{~V} 4 \mathrm{M} 5)$ at the $9^{\text {th }}$ week. At the end of $9^{\text {th }}$ week, the patient was able to communicate fully with GCS 12 (E3V4M5) and was transferred from HDU to Physical Medicine Department for rehabilitation. Clinically, the patient was functionally independent at $11^{\text {th }}$ week. Follow up MRI of brain showed significant resolution of parieto-occipital lesion after week 9 (Figure 2).

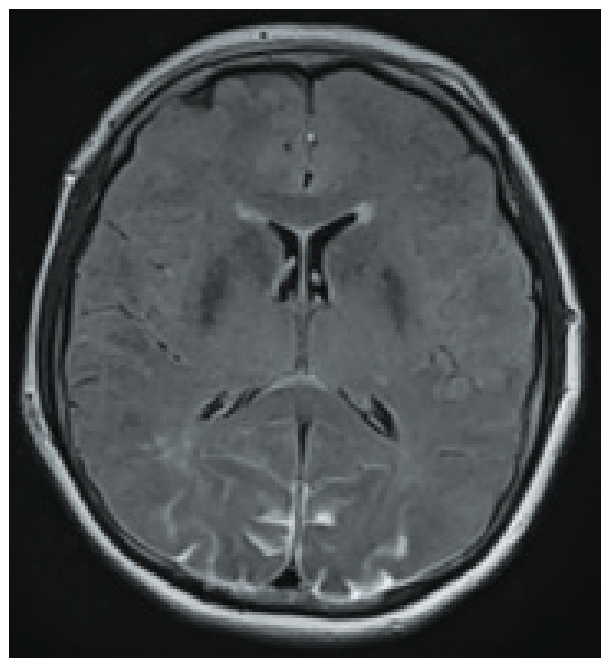

Figure 2 Follow up MRI of brain (FLAIR) showing marked decrease of hyper-intensity in parieto-occipital region (at $9^{\text {th }}$ week)

\section{Discussion}

PRES is a reversible condition which involves mainly the posterior part of the brain. ${ }^{1,2}$ PRES was first reported and described in 1996 as a reversible encephalopathy. 2,5 This syndrome was first reported as reversible posterior leukoencephalopathy syndrome (RPLS) which was renamed as PRES in the year 2000. ${ }^{2,6}$ Although the mechanism or pathogenesis of this reversible syndrome is still not clear, brain autoregulation mechanism and cerebral blood vessel endothelium dysfunction may have key role for this disease. ${ }^{1,6}$ Sudden hypertensive episode can lead to failure of cerebral autoregulation mechanism. This causes acute disruption of blood brain barrier which causes reversible cerebral edema. ${ }^{6}$

MRI of brain can be the diagnostic imaging modality for PRES. T2-weighted images andfluid attenuated inversion recovery (FLAIR) images in MRI can detect the vasogenic cerebral oedema by hyper-intense signal change at posterior subcortical region. ${ }^{7}$ Parietal and occipital lobes are commonly involved in PRES. But, 
presented with altered consciousness for an unusual longer period of time ( 7 weeks) and eventually recovered both clinically and radiologically. Although the recovery in our case was incomplete due to multiple risk factors (prolonged stress hyperglycemia and pulmonary infection) and very long disease course, the reversibility was marked after a period of no response.

The overall outcome of PRES depends on abnormalities in imaging like infarction, hemorrhage and brain edema (mainly vasogenic edema). ${ }^{8}$ Hyperglycemia is an important factor for poor outcome in PRES. ${ }^{2,8}$ Septicemia and dementia can also play significant role in the overall outcome in PRES. ${ }^{9}$ The lesion size in MRI corresponds with the overall outcome in PRES. ${ }^{9}$ In our case, multiple factors like hyperglycemia, chest infection and large lesion size (parietal and occipital lobe) might be the reason for the delayed recovery.

Complete recovery is found in more than $50 \%$ cases in PRES. The mortality is high $(15 \%)$ if the treatment is delayed or inadequate. ${ }^{8,9}$ In severe PRES, the reversibility can be complete if diagnosed early, treated and rehabilitated correctly. ${ }^{9,10}$ Underdiagnosed or misdiagnosed cases can lead to massive ischemic changes in the brain which is irreversible and carries poor prognosis. ${ }^{10}$

\section{Conclusion}

It is absolutely crucial to diagnose and treat PRES early because of its complete reversible outcome. As reversibility can be delayed for more than 2 months, high index suspicion and long term follow up care with multidisciplinary specialty team is necessary. Preventing poor prognostic indicators can ensure good outcome. Further clinical and radiological evaluation is needed for better understanding the mechanism, treatment modalities and prognosis of atypical, delayed reversal cases of PRES.

Conflict of interest: Nothing to declare.

\section{References}

1. Bartynski WS. Posterior reversible encephalopathy syndrome, part 2: controversies surrounding pathophysiology of vasogenic edema. Am J Neuroradiol 2008;29:1043-44.

2. Fischer M, Schmutzhard E. Posterior reversible encephalopathy syndrome. J Neurol 2017;264:1608-16.

3. Zhang L, Wang Y, Shi L, Cao J, Li Z, Wáng YX. Late postpartum eclampsia complicated with posterior reversible encephalopathy syndrome: a case report and a literature review. Quant Imaging Med Surg 2015;5:909-16.

4. Ogura, S, Narumiya, H, Iiduka, R, Nagakane Y. Late recovery from unconsciousness in a patient with severe posterior reversible encephalopathy syndrome. Clinical Case Reports 2008;6:1825-28.

5. Brewer J, Owens MY, Wallace K, Reeves AA, Morris R, Khan $\mathrm{M}$, et al. Posterior reversible encephalopathy syndrome in 46 of 47 patients with eclampsia. Am J ObstetGynecol 2013;208:468.e1-6.

6. Vaughan CJ, Delanty N. Hypertensive emergencies. Lancet 2000;356:411-17.

7. Bartynski WS, Boardman JF. Distinct imaging patterns and lesion distribution in posterior reversible encephalopathy syndrome. Am J Neuroradiol 2007;28:1320-27.

8. Liman TG, Bohner G, Endres M, Siebert E. Discharge status and in hospital mortality in posterior reversible encephalopathy syndrome. ActaNeurolScand 2014;130: 34-39.

9. Legriel S, Schraub O, Azoulay E. Determinants of recovery from severe posterior reversible encephalopathy syndrome. PLoS ONE 2012;7:e44534.

10. Stevens CJ, Heran MK. The many faces of posterior reversible encephalopathy syndrome. Br J Radiol2012;85:1566-75. 